CANCER

\section{Environment of chemo success}

A tumour's response to chemotherapy is shaped by interactions between the tumour and its microenvironment.

Mikala Egeblad at Cold Spring Harbor Laboratory in New York and her colleagues used in vivo microscopy to monitor tumours' responses to the chemotherapy drug doxorubicin in mice. They found that the drug is more effective in a mouse model of breast cancer with intermediate tumours rather than precancerous changes or late-stage tumours, and in animals that lack the enzyme MMP9, which acts on the protein matrix surrounding cells and tumours.

The improved drug response seemed to be linked to increased leakage from tumour blood vessels, which facilitates drug access. Moreover, mice lacking a receptor called CCR2 responded more strongly to doxorubicin than did mice with the receptor. Immune cells that express the CCR2 receptor are attracted to tumours as a result of doxorubicin treatment - this can promote tumour regrowth.

Drugs that inhibit MMP9 and CCR 2 could be combined with traditional chemotherapies to boost cancer treatment success, the authors suggest.

Cancer Cell 21, 488-503 (2012)

\section{ENVIRONMENTAL HEALTH}

\section{Less biodiversity, more allergies}

A decrease in the amount of time spent in contact with the natural environment and changes in the population of microbes resident on the

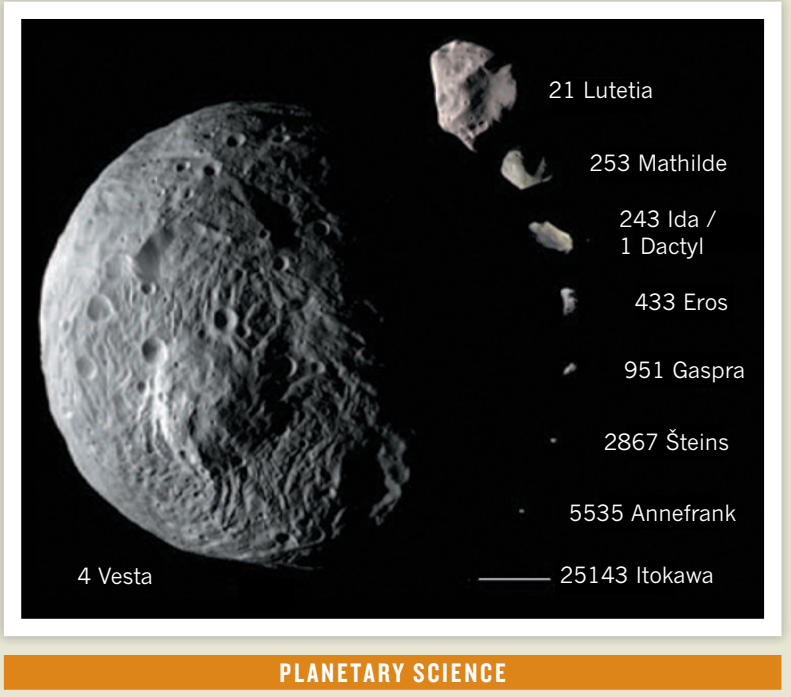

\title{
Planet-like asteroid
}

The giant asteroid Vesta resembles a planet more than it does other asteroids, according to Christopher Russell at the University of California, Los Angeles, and his colleagues. In six separate studies, the researchers report their analysis of data from NASA's Dawn spacecraft, which has been orbiting Vesta (pictured, relative to other asteroids) since July 2011.

Vesta was formed about 2 million years after the Solar System's first solid bodies and is the Solar System's secondlargest asteroid. The authors report that Vesta is pockmarked with many impact craters, including two overlapping ones, several hundred kilometres wide, at the south pole. One of these polar impacts blasted off material that became more asteroids, known as Vestoids, and meteorites. Shocks from these two big impacts apparently created the troughs that ring Vesta's equator. The asteroid's large size - roughly 260 kilometres in radius - rapid growth and massive iron core may explain how Vesta survived all these collisions.

Science 336, 684-686; 687-690; 690-694; 694-697; 697-700; 700-704 (2012)

skin could be contributing to the increase in inflammatory disorders such as allergies.

To test these ideas, Ilkka Hanski at the University of Helsinki and his colleagues measured immune reactions to common allergens and the composition of skin microbes in 118 adolescents in eastern Finland. The team used the mixture of plants in the young peoples' gardens along with local land use as measures of biodiversity. They found that people living in areas of reduced biodiversity were more prone to allergies, and that allergic individuals had distinct populations of bacteria on their skin. Among healthy individuals, those with a greater abundance of the bacterial genus Acinetobacter on the skin produced higher levels of the immunoregulatory protein IL-10, which helps the body to tolerate harmless substances.
Loss of biodiversity could cause problems for public health as well as for the environment, the researchers suggest.

Proc. Natl Acad. Sci. USA http://dx.doi.org/10.1073/ pnas.1205624109 (2012)

\section{Anti-seizure drug boosts memory}

One way to improve memory in people with a disorder that can precede Alzheimer's disease is to dampen activity in a part of the brain known as the hippocampus, rather than to boost it as previously thought.

Michela Gallagher at Johns Hopkins University in Baltimore, Maryland, and her colleagues tested the memory of patients with amnestic mild cognitive impairment - in which a person's memory is worse than expected for their age - following treatment with a low dose of an anti-seizure drug, levetiracetam, which dampens excess hippocampal activation. Patients given the drug made fewer mistakes on a memory task and showed less hippocampal activity in brain scans than those given a placebo.

Regulating neural activity could control the progression of Alzheimer's disease, the authors suggest.

Neuron 74, 467-474 (2012)

\section{BIOMATERIALS}

\section{High-voltage plant proteins}

Crystals of photosynthetic protein complexes extracted from plant cells can generate extraordinarily high voltages when placed on a conducting surface and stimulated by light.

Each of the lighttransducing complexes known as photosystem I 